\title{
Minimal Revenue Tolls: Price Stability for Networks With BPR Cost Function
}

\author{
Claude M. Penchina*
}

Department of Physics, University of Massachusetts, Amherst, MA 01003, USA;

Department of Physics, King's College, Strand, London WC2R-2LS, UK;

ECE Department, UCSD, La Jolla CA 92093, USA; Gilora Associates, Flemington, NJ, USA;

CE Department, San Diego State University, San Diego, CA 92182, USA

\begin{abstract}
Marginal-Cost (MC) tolls are known to produce economic efficiency of network flows. Yet, MC pricing has not been widely adopted, because of various perceived unpopular aspects, such as complexity, collection costs, and inequities. Minimal Revenue (MinRev) pricing has been suggested as a means to achieve most of the economic improvements of MC pricing with fewer unpopular aspects. One claimed improvement of MinRev tolls is the ability to maintain fixed tolls while flows change. We show that single-power-law congestion costs are sufficient (but not always necessary) conditions for the stability (flow independence) of Minimal-Revenue (MinRev) tolls in transportation networks, so long as the links that are actually used do not change. This is particularly important because the usual Bureau of Public Roads (BPR) cost function recommended as a good representation of real road traffic, has this single power-law congestion cost. For cases of elastic demand, these MinRev tolls do not achieve the full economic efficiency of MarginalCost (MC) tolls. However, they may still prove desirable because of greater stability, greater simplicity, lower out-ofpocket costs, and greater perceived equity. Furthermore, in cases of major congestion problems, where all links are used, the MinRev tolls are totally stable, i.e. totally independent of flows; also, if demand is relatively insensitive to price, the flows are nearly equal to the optimal flows obtained by MC pricing. The discussion is based on the equilibrium assignment method, with tolls to encourage System Optimal (SO) flows rather than User Optimal (UO) flows. Results are obtained for general networks, and also illustrated with a very simple example.
\end{abstract}

Keywords: BPR, minimal revenue pricing, marginal cost pricing, road traffic, traffic congestion, toll, travel time, capacity, traffic assignment, example, optimization.

Mots-clés Français/French Keywords: BPR, Fixation de prix de Revenu Minimale, Fixation de prix de Prix Marginale, Trafic routier, Congestion trafic, Péage, Durée trajet, Capacité, Affectation trafic, Exemple, Optimisation.

Mots-clés Espagnols/Spanish Keywords: BPR, Fijación de precios de Ingresos Mínima, Fijación de precios de Coste Marginal, Tráfico carretera, Congestión tráfico, Peaje, Duración trayecto, Capacidad, Afectación tráfico, Ejemplo, Optimización.

\section{INTRODUCTION}

Generalized-costs, combinations of travel times and costs (direct monetary and other), consist of fixed costs and congestion costs. For a particular road link, the fixed costs are due to the length of the road, speed limits, etc., when the link is un-congested. The congestion costs are the additional time and costs caused by the congestion of other users. The congestion cost functions used in transport network analysis, are often modeled as simple powers of the flow as recommended by the Bureau of Public Roads to represent real traffic network flows; the so-called BPR cost function [1] (See appendix 1). 1st power (linear) [2,3] \{see p.140 of ref 3$\}, 2$ nd power (quadratic) [3] \{see p. 91 of ref 3$\}$, and 4th power [3-5] \{see p. 127 of ref 3$\}$ are popular choices.

*Address correspondence to this author at the Department of Physics, University of Massachusetts, Amherst, MA 01003, USA; Tel: 413-5452545; E-mails: penchina@physics.umass.edu, cmpenchina@gmail.com
In the equilibrium traffic assignment problem, MarginalCost (MC) pricing is a well-known means to induce network users to "voluntarily" adjust the flow pattern to achieve economic efficiency [4-6]. Each user is charged for the additional system costs incurred because of his use of the link. This induces users to optimize the flow pattern, and minimizes the total system costs (not counting the tolls). MC tolls often involve large unpopular out-of-pocket costs to the users. Thus, although MC pricing has economic efficiency, it is not used very much in practice because of several perceived negative aspects. It is perceived as inequitable since it allows users who can afford the tolls (presumably those with a higher monetary value of time: often the richer users, but also those trying to catch an airplane with nonrefundable tickets, etc.) to travel faster than other (often poorer) users. (See appendix 2). The MC tolls are also "unstable" in the sense that they must be varied with time as the congestion varies, an aspect that adds complexity and cost to the collection system and unpredictability to the user. 
The idea of Minimal-Revenue (MinRev) pricing is to lower tolls as much as possible, without upsetting the optimum flow pattern that would be achieved by $\mathrm{MC}$ pricing. MinRev tolls have been suggested as a means to obtain the same economic efficiency with much lower outof-pocket costs to the users, and simpler collection procedures, with fewer links needing tolls [4, 5]. Dial illustrated his MinRev algorithm with a numerical network example. We illustrate the MinRev pricing with the much simpler and smaller network of Fig. (1), which is easily solved by inspection. By eliminating or reducing some of the negative aspects of MC pricing, it is hoped that MinRev pricing will be implemented more often than $\mathrm{MC}$, while still achieving most of the advantages of $\mathrm{MC}$ pricing. MinRev pricing may be particularly applicable to paid so-called "HOT" lanes on freeways. (See appendix 2).

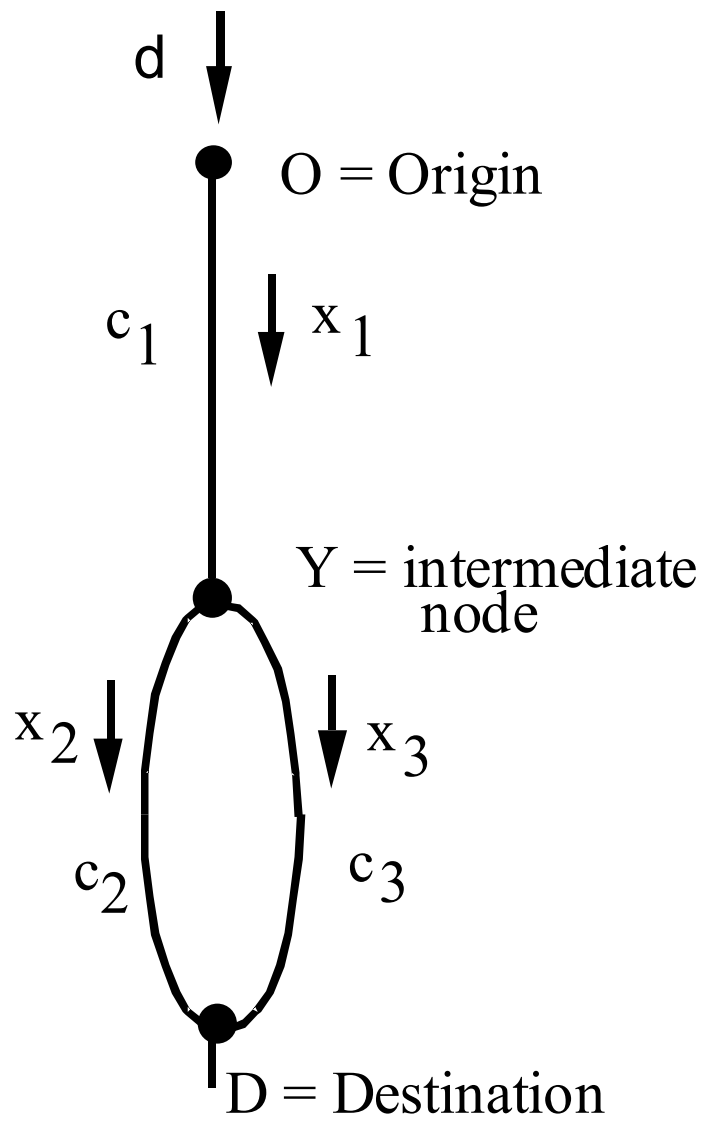

Fig. (1). A simple network with 3-links, 2-paths, 1-origin O, and 1destination D, used to illustrate User-Optimal (UO), and SystemOptimal (SO) flows with Marginal Cost (MC) tolls and Minimum Revenue (MinRev) tolls.

\section{Symbols used in this figure:}

d demand

$\mathrm{x}_{\mathrm{j}} \quad$ flow $\mathrm{x}$ on link $\mathrm{j}$

arrows show direction of flow

$\mathrm{h}_{\mathrm{j}}$ Fixed Cost $\mathrm{h}$ on link $\mathrm{j}$

$\left(\mathrm{x}_{\mathrm{j}}\right)^{\mathrm{n}} \mathrm{g}_{\mathrm{j}} \quad$ Congestion cost on link $\mathrm{j}$

$c_{j}=h_{j}+\left(x_{j}\right)^{n} g_{j} \quad$ Total user generalized cost $\mathrm{c}$ on link $j$

$\mathrm{j}=1,2,3$
Although MinRev tolls achieve full economic efficiency for cases of inelastic demand, they don't for elastic demand [7]. Still, as we shall see, MinRev tolls may often be a desirable alternative to MC tolls because of fewer perceived negative aspects, and very small deviations from full efficiency. We find that there are certain conditions under which MinRev tolls are piecewise stable, i.e. totally independent of flow, so long as the links actually used in the network do not change, and then a step change when the used links do change. In cases where there are major congestion problems and all links are used, i.e. for cases in which one would most want to use tolls to alleviate the congestion, we find that the MinRev tolls may be totally stable (independent of changes in flow), and the flows may be nearly optimal. Thus deviations from full economic efficiency may be insignificant. The advantages of the MinRev pricing could then often outweigh the disadvantage of slight deviations from efficient flows.

\section{AND MINREV PRICING FOR INELASTIC DEMAND}

MinRev pricing lowers the MC tolls as much as possible, without upsetting the optimum flows that would be achieved by MC pricing. Thus, by its very design MinRev pricing achieves the same full economic efficiency (by optimizing flows) as MC pricing. Often this can be achieved with much lower out-of-pocket costs. Dial's algorithms to achieve MinRev pricing limit tolls to non-negative values, and yield the minimum total system revenue that does not upset the optimum flows. This often leads to several links (and always at least one $[4,5]$ ) on which no toll is collected. We note also that, if one allows negative tolls (subsidies), it is possible to achieve a neutral (net zero) total revenue. However, this would eliminate the advantage of fewer links needing tolls.

\section{MC and MinRev Pricing for Power-Law Congestion Costs}

We consider the generalized link costs $c_{j}$ of the form

$\mathrm{c}_{\mathrm{j}}=\mathrm{h}_{\mathrm{j}}+\left(\mathrm{x}_{\mathrm{j}}\right)^{\mathrm{n}} \mathrm{g}_{\mathrm{j}}$

Here, for link $\mathrm{j}, \mathrm{x}_{\mathrm{j}}$ is the flow, $\mathrm{h}_{\mathrm{j}}$ is the fixed cost, and $\left(x_{j}\right)^{n} g_{j}$ is a power law congestion cost with power $n$. $g_{j}$ is a constant, which depends on link $\mathrm{j}$, but the power $\mathrm{n}$ is the same on each link.

The Marginal Cost Toll $\left(\mathrm{Tmc}_{\mathrm{j}}\right)$, on link $\mathrm{j}$ is

$\mathrm{Tmc}_{\mathrm{j}}=\mathrm{x}_{\mathrm{j}} \partial \mathrm{c}_{\mathrm{j}} / \partial \mathrm{x}_{\mathrm{j}}=\mathrm{n}\left(\mathrm{x}_{\mathrm{j}}\right)^{\mathrm{n}} \mathrm{g}_{\mathrm{j}}$

and $\mathrm{Cmc}_{\mathrm{j}}$, the Generalized Cost of link $\mathrm{j}$ with $\mathrm{MC}$ toll, becomes

$\mathrm{Cmc}_{\mathrm{j}}=\mathrm{c}_{\mathrm{j}}+\mathrm{Tmc}_{\mathrm{j}}=\mathrm{h}_{\mathrm{j}}+(\mathrm{n}+1)\left(\mathrm{x}_{\mathrm{j}}\right)^{\mathrm{n}} \mathrm{g}_{\mathrm{j}}$

It will be useful later to note that

$\left(\partial \mathrm{Tmc}_{\mathrm{j}} / \partial \mathrm{x}_{\mathrm{j}}\right) /\left(\partial \mathrm{Cmc}_{\mathrm{j}} / \partial \mathrm{x}_{\mathrm{j}}\right)=\mathrm{n} /(\mathrm{n}+1)$

is a constant, independent of $\mathrm{j}$.

\section{Zero Fixed Costs}

For the special case where the fixed costs are all zero, Eqs. (1) and (3) become

$\mathrm{h}_{\mathrm{j}}=0 \quad \mathrm{c}_{\mathrm{j}}=\left(\mathrm{x}_{\mathrm{j}}\right)^{\mathrm{n}} \mathrm{g}_{\mathrm{j}}$

$\mathrm{h}_{\mathrm{j}}=0 \quad \mathrm{Cmc}_{\mathrm{j}}=(\mathrm{n}+1)\left(\mathrm{x}_{\mathrm{j}}\right)^{\mathrm{n}} \mathrm{g}_{\mathrm{j}}=(\mathrm{n}+1) \mathrm{c}_{\mathrm{j}}$ 
We see that the toll multiplies the generalized cost of each link by the same constant factor $n+1$. By symmetry, we see that with fixed demand, the MC pricing does not change the flow pattern in the network. Thus, one already has the optimized flow without any link tolls, so the MinRev tolls are all zero, independent of the flow, so:

Conclusion (1) For single-power law congestion costs and zero fixed costs, all MinRev tolls are stable at a value of zero, independent of the flow. Thus the flows are optimal without tolls.

Although this conclusion (1) is interesting theoretically, and applicable to various physical networks, it has very little applicability in real traffic networks since real road links always have some fixed costs (although those costs could be compensated by subsidies or scenic views, etc). It is, nonetheless, useful as a step towards proving the stability of MinRev tolls when the fixed costs are not zero.

\section{Non-Zero Fixed Costs}

The simple example of Fig. (1) provides much insight into the MinRev tolls. It has only three links and two paths from the origin $\mathrm{O}$ to the destination $\mathrm{D}$. It is seen by inspection that both paths must use link 1. Thus, for inelastic demand $\mathrm{d}$, the flow through link 1 is $\mathrm{x}_{1}=\mathrm{d}$.

This flow cannot be influenced by any toll, even if the cost function is much more complicated than that of Eq. (1). The MinRev toll on this link, being the minimum needed to assure optimal flows, is clearly zero, independent of the (inelastic) network demand or the relationship between cost and flow in this link. So:

Conclusion (2) There is no necessary condition for stable MinRev tolls.

Consider now links 2 and 3 of Fig. (1). It is clear that only the difference between the tolls on these links can influence the relative flows. This leads us to an algorithm for MinRev tolls: the smaller MC toll should be replaced by zero toll, and the larger one replaced by the difference between the two. Thus, we find that only one toll is needed on only one link in the network of Fig. (1). This is an example of Dial's result that MinRev always has at least one zero-toll path.

For simplicity of the discussion, and without loss of generality, we let the MC toll (Tmc) be smaller on link 2 than on link 3. The MinRev toll on link $3\left(\mathrm{TMinRev}_{3}\right)$ is then

$\mathrm{TMinRev}_{3}=\mathrm{Tmc}_{3}-\mathrm{Tmc}_{2}$

We now examine the stability of $\mathrm{TMinRev}_{3}$ under changes of inelastic demand $d$. So long as the number of used links does not change, the variation of $\mathrm{TMinRev}_{3}$ with demand $d$ will be smooth, so we can use calculus to examine the changes.

$\mathrm{TMinRev}_{3}=\mathrm{Tmc}_{3}-\mathrm{Tmc}_{2}=\mathrm{n}\left\{\left(\mathrm{x}_{3}\right)^{\mathrm{n}} \mathrm{g}_{3}-\left(\mathrm{x}_{2}\right)^{\mathrm{n}} \mathrm{g}_{2}\right\}$

From Kirchhoff's current law,

$\mathrm{x}_{3}+\mathrm{x}_{2}=\mathrm{d}$

If only one path is used: If $x_{2}=0$, the results are simple, and the MinRev tolls are all zero and thus all stable. $\mathrm{x}_{3}$ cannot be zero unless $\mathrm{d}=0$, which is trivial and uninteresting.
If both paths are used: By Wardrop's first principle [8] (or Kirchhoff's voltage law) the user costs $\mathrm{Cmc}_{\mathrm{j}}$ including $\mathrm{MC}$ tolls are equal for links 2 and 3.

$\mathrm{Cmc}_{\mathrm{j}}=\mathrm{c}_{\mathrm{j}}+\mathrm{Tmc}_{\mathrm{j}}$

$\mathrm{Cmc}_{3}=\mathrm{Cmc}_{2}$

This must be true for any level of inelastic demand $d$. Thus, if the demand d changes, by differentiating $\mathrm{Eq}(8)$, we get

$\partial \mathrm{Cmc}_{3} / \partial \mathrm{d}=\partial \mathrm{Cmc}_{2} / \partial \mathrm{d}$

so

$\left\{\partial \mathrm{Cmc}_{3} / \partial \mathrm{x}_{3}\right\}\left\{\partial \mathrm{x}_{3} / \partial \mathrm{d}\right\}=\left\{\partial \mathrm{Cmc}_{2} / \partial \mathrm{x}_{2}\right\}\left\{\partial \mathrm{x}_{2} / \partial \mathrm{d}\right\}$

and

$\left\{\partial \mathrm{x}_{2} / \partial \mathrm{d}\right\}=\left\{\partial \mathrm{x}_{3} / \partial \mathrm{d}\right\}\left\{\partial \mathrm{Cmc}_{3} / \partial \mathrm{x}_{3}\right\} /\left\{\partial \mathrm{Cmc}_{2} / \partial \mathrm{x}_{2}\right\}$

When inelastic demand d changes, from $\mathrm{Eq}(5)$

$\partial \mathrm{TMinRev}_{3} / \partial \mathrm{d}=\partial \mathrm{Tmc}_{3} / \partial \mathrm{d}-\partial \mathrm{Tmc}_{2} / \partial \mathrm{d}$

$=\left\{\partial \mathrm{Tmc}_{3} / \partial \mathrm{x}_{3}\right\}\left\{\partial \mathrm{x}_{3} / \partial \mathrm{d}\right\}-\left\{\partial \mathrm{Tmc}_{2} / \partial \mathrm{x}_{2}\right\}\left\{\partial \mathrm{x}_{2} / \partial \mathrm{d}\right\}$

From Eq (11) we find

$\partial \mathrm{TMinRev}_{3} / \partial \mathrm{d}=$

$\left\{\partial \mathrm{Cmc}_{3} / \partial \mathrm{x}_{3}\right\}\left\{\partial \mathrm{x}_{3} / \partial \mathrm{d}\right\}$

$\left[\left(\left\{\partial \mathrm{Tmc}_{3} / \partial \mathrm{x}_{3}\right\} /\left\{\partial \mathrm{Cmc}_{3} / \partial \mathrm{x}_{3}\right\}\right)-\left(\left\{\partial \mathrm{Tmc}_{2} / \partial \mathrm{x}_{2}\right\} /\left\{\partial \mathrm{Cmc}_{2} / \partial \mathrm{x}_{2}\right\}\right)\right]$

From Eq (4) we see that the term in square brackets [ ] is zero. Thus,

$\partial \mathrm{TMinRev}_{3} / \partial \mathrm{d}=0$

so in this example network, the MinRev toll is totally stable for all flows whenever both paths are used.

Summary for the example network of Fig. (1): A MinRev toll is needed only on link 3. This $\mathrm{TMinRev}_{3}$ is piecewise stable. $\mathrm{TMinRev}_{3}=0$ for all demands small enough for only one path to be used, and then constant at value $\mathrm{TMinRev}_{3}=\mathrm{Tmc}_{3}-\mathrm{Tmc}_{2}$ when both paths are used. The proof of stability of the tolls is easily extended to any integer number $m$ (not just 2) of links in parallel. This result also leads us to conjecture a new conclusion, which we then prove for a general network:

Conclusion (3) A cost function $c_{j}=h_{j}+g_{j}\left(x_{j}\right)^{n}$ on each link $\mathbf{j}$ of a general traffic network, is sufficient (but not always necessary) for piecewise stable MinRev tolls. The tolls may change from one stable value to another as the links used in the network change when the demand changes.

Proof of Conclusion (3) We consider a general network, with power-law congestion costs as in Eq (1), in which we already have equilibrium with MinRev tolls. If demands then change, the link flows $\mathrm{x}_{\mathrm{j}}$ change, and the corresponding $\mathrm{MC}$ tolls also change, as in Eq (3). For a nearby range of demands over which the list of links which are actually used does not change, we expect smooth changes in flows and costs, so we can use differential calculus for our analysis of corresponding changes in MinRev tolls, which will turn out to be zero for a fixed integer number of used links. Differentiating Eq (8) with respect to flows, for the link costs $\mathrm{Cmc}_{\mathrm{j}}$ including $\mathrm{MC}$ tolls, we find 
$\partial \mathrm{Cmc}_{\mathrm{j}} / \partial \mathrm{x}_{\mathrm{j}}=\partial \mathrm{c}_{\mathrm{j}} / \partial \mathrm{x}_{\mathrm{j}}+\partial \mathrm{Tmc_{j }} / \partial \mathrm{x}_{\mathrm{j}}$

and using Eqs (1) and (2), we find

$\partial \mathrm{c}_{\mathrm{j}} / \partial \mathrm{x}_{\mathrm{j}}=\mathrm{n}\left(\mathrm{x}_{\mathrm{j}}\right)^{\mathrm{n}-1} \mathrm{~g}_{\mathrm{j}}$

$\partial \mathrm{Tmc}_{\mathrm{j}} / \partial \mathrm{x}_{\mathrm{j}}=\mathrm{n}^{2}\left(\mathrm{x}_{\mathrm{j}}\right)^{\mathrm{n}-1} \mathrm{~g}_{\mathrm{j}}$

so

$\partial \mathrm{Cmc}_{\mathrm{j}} / \partial \mathrm{x}_{\mathrm{j}}=\mathrm{n}(\mathrm{n}+1)\left(\mathrm{x}_{\mathrm{j}}\right)^{\mathrm{n}-1} \mathrm{~g}_{\mathrm{j}}$

The derivatives of $\mathrm{c}_{\mathrm{j}}, \mathrm{Tmc}_{\mathrm{j}}$, and $\mathrm{Cmc}_{\mathrm{j}}$, are independent of the $h_{j}$, so that changes in costs are identical to those which would be obtained with $h_{j}=0$, for which the flows were shown to be optimal. The increases in link flows due to increases in demand are thus also independent of $h_{j}$ and are the same optimal extra flows with or without tolls, just as the total flows were optimal for all $h_{j}=0$. Thus, optimality of flows applies to the extra demand here, just as it applies to the total demand for zero fixed costs. Just as the total MinRev tolls were all zero for zero fixed costs, here the extra MinRev tolls are zero for non-zero fixed costs. Thus, the MinRev tolls are stable until the demands increase enough to change which of the links are used, causing a discontinuous change in flows and invalidating our use of the differential calculus. From there on, the MinRev tolls are again stable at a new level as long as the same links are used, so the $\mathrm{TMinRev}_{\mathrm{j}}$ are Piecewise Constant, completing the proof.

Summary for inelastic demand and power law congestion costs: We used the ideas of optimality, to prove the stability of Minimal Revenue MinRev tolls in networks with inelastic demands. We found it also helpful to study a simple example network. We found that link costs which include a fixed cost and single-power-law congestion cost are sufficient (but not always necessary) to cause MinRev tolls to be piecewise stable, remaining constant when the used links remain unchanged, and then jumping discontinuously when the used links change.

It is especially important to note that the so-called BPR cost function recommended by the Bureau of Public Roads Traffic Assignment Manual [1] does have a fixed cost and a single-power-law congestion cost. Thus, power-law congestion costs are important examples for real road networks, not just the simple example used in this paper and in previous MinRev examples [4,5].

If the fixed costs are zero on all links, then the MinRev tolls are zero (totally stable) for all levels of inelastic demand. This is a rather unrealistic situation for real road networks, since the free-flow generalized costs would have to be zero on each link. It could happen, even with positive travel times, if there are exactly compensating other negative costs (such as popular scenery, carefully adjusted monetary subsidies, etc). It may be a more realistic approximation for various other analogous physical networks (electrical, mechanical, and thermal [9-11] or water [12]), but then the analog of tolls is not obvious. A more clearly applicable analog might be a data network.

One should note that generally, congestion tolls are proposed and/or used only in cases where there are major congestion problems. Then, it is very likely that the total demand is so high that all links are used. In that case, the MinRev tolls are totally stable.

\section{AND MINREV PRICING FOR ELASTIC DEMAND}

For demands with price-elasticity, MC pricing does not only induce a rearrangement of the flows among the links. The increased total cost due to MC tolls also causes a reduction in the total network flow, resulting in a true economic efficiency. On the other hand, the algorithms used for MinRev tolls [4, 5] are based on a particular level of flow, and do not achieve the optimality of MC tolls, because their lower costs cause a smaller reduction in total flow. However, they do have the advantages of stability, and lower-total system revenue, and are thus perceived as more equitable and less unpredictable [4, 5]. Hence, MinRev may still be the preferred pricing method if elasticity of demand is not very important.

The fact that rush hour congestion is often a major problem in real road networks, is an indication that the number of users remains large in spite of the high generalized cost caused by the congestion. This indicates that the high demand is often not very sensitive to price. MC pricing would then improve flows mainly by changing the road assignment without much change in the total flows. MinRev pricing will do almost the same, and thus also provide nearly optimal flows under changes of demand that are nearly price-inelastic. Furthermore, with such large flows, it is likely that all links in the network are actually used, leading to MinRev tolls that are totally stable, independent of time dependent demands.

\section{DISCUSSION AND CONCLUSIONS}

Minimal Revenue (MinRev) pricing has been known to have various advantages over Marginal Cost (MC) pricing in road networks. The tolls are lower, the number of links needing tolls is smaller, and the tolls are more stable under conditions of time varying demands. These advantages lead to lower collection costs, less user confusion, and more perceived equitability $[4,5]$.

We have shown that the existence of single-power-law congestion costs is a sufficient (although not always necessary) condition for piecewise stable MinRev tolls; the tolls do not change so long as there is no change in the links which are used. This leads to a total stability of tolls (no change with flow) so long as the total demand is sufficiently large that all links are used.

Single-power-law congestion cost is commonly used as a representation of actual conditions, as suggested by the Bureau of Public Roads recommendation [1].

We note that the cases of most interest for congestion pricing are those for which the demand remains so high, even with the high congestion, that it is very likely that all network links are actually used and that the demand is relatively price-inelastic. Under such conditions, the MinRev pricing becomes totally stable (independent of time varying flows), and the flows are nearly optimal. MinRev pricing also retains the advantage of lower out-of-pocket costs to the users, thus mediating the perception that tolls are inequitable and a regressive form of taxation. These advantages should make MinRev pricing a very attractive alternative to $\mathrm{MC}$ pricing in most cases where tolls are considered necessary to relieve congestion. 
It would be interesting to test the MinRev method in actual traffic situations, to observe if it really works almost as well as the MC method during rush hours, and to determine if the users perceive it as better for reasons of equitability, lower out of pocket costs, etc. As pointed out by an anonymous reviewer, it would also be interesting to study the effects of MR pricing in situations with oversaturation and queue growth.

\section{ACKNOWLEDGEMENTS}

We wish to thank Bruce Calvert, Robert Dial, Grant Keady, and Robin Lindsey for several very useful discussions. We thank James Banks and Benjamin Heydecker for information about the Bureau of Public Roads.

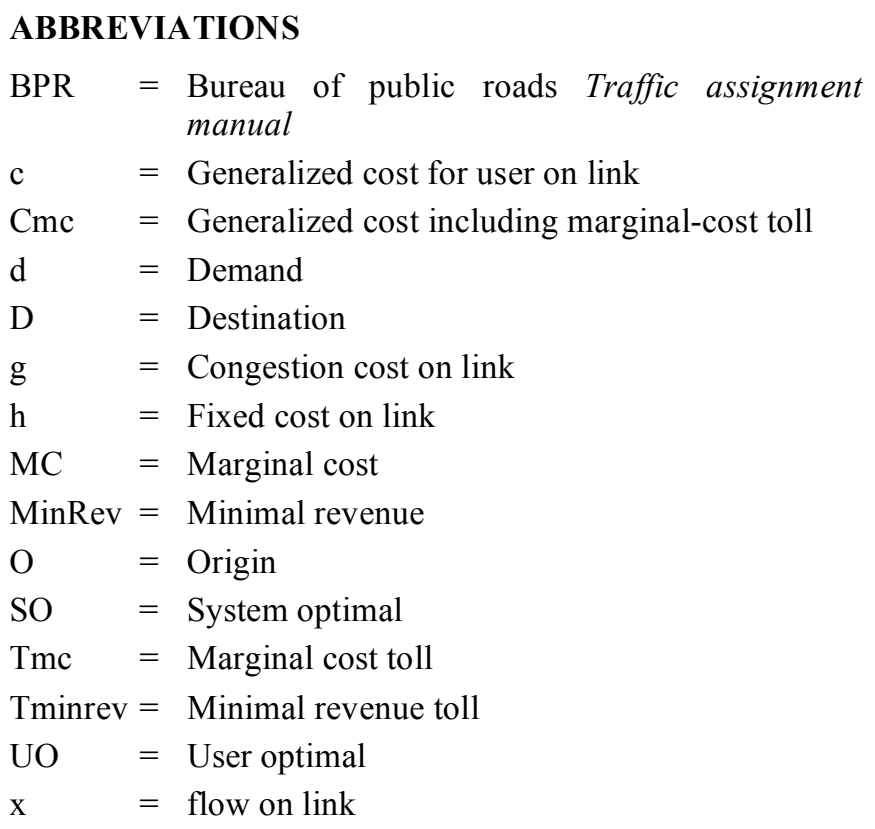

\section{APPENDIX 1 - BUREAU OF PUBLIC ROADS (BPR) COST FUNCTION [13]}

The Bureau Of Public Roads (1964) Traffic assignment manual [1] recommended a cost versus flow function of the form

$$
\mathrm{t}(\mathrm{x})=\mathrm{t}(0)\left[1+\mathrm{a}(\mathrm{x} / \mathrm{Q})^{\mathrm{b}}\right]
$$

where $\mathrm{x}$ is the flow

$t(x)$ is the travel time when the flow is $x$

$t(0)$ is the free-flow travel time, when $x$ is near zero.

$\mathrm{Q}$ is the capacity of the road link

$\mathrm{a}, \mathrm{b}$ are parameters.

The original manual included a table of recommended parameter values $a$ and $b$ for different kinds of roads. The most common values now used are $a=0.15$ and $b=4$ (hence the oft used misnomer "fourth power law"). This fourth power law was used by Dial in the demonstration examples for his algorithm $[4,5]$.
Comparison with our Eq. (1) shows that:

$\mathrm{t}(\mathrm{x})$ corresponds to our generalized cost, $\mathrm{c}$.

$\mathrm{t}(0)$ corresponds to our fixed cost, $h$.

The product $\left\{\mathrm{t}(0) \mathrm{a} \mathrm{Q}^{-\mathrm{b}}\right\}$ corresponds to our congestion cost per unit flow, $g$

b corresponds to our power, $n$.

\section{APPENDIX 2 - IN-EQUITABILITY OF MARGINAL COST (MC) PRICING}

In equilibrium, all used paths between an origin and destination have equal generalized costs. Thus, those with higher monetary costs (e.g., higher tolls) will have lower other costs (often time). Thus, it is claimed that those who are willing to pay the tolls will travel faster (or at least have lower other generalized costs) than those who are not willing to pay the tolls. The time saving is particularly true for socalled "HOT" lanes, toll lanes competing with parallel free lanes on the same multilane highway, where all generalized costs other than tolls and time would be equal.

Actually, with tolls, even those users who do not pay the tolls will travel faster than before because of the improvements in flow produced by the toll policy. However, this is not as easily noticed as the inequity of seeing others paying the toll and going still faster.

Those willing to pay the toll presumably have a higher money-value of time. These may include road users with high-cost non refundable tickets to a cultural event or airline flight, high costs of being late to work, etc. However, they may also include richer users who can more easily afford the tolls than poorer users. This latter effect of diversity is usually perceived to be somewhat inequitable.

In any case, the tolls represent a larger fraction of income for poorer users (presumably many who can also not afford to be late to work). This is seen as an unpopular regressive form of taxation, a perception which may be hard to avoid.

\section{REFERENCES}

[1] BPR, Bureau of Public Roads Traffic Assignment Manual: U.S. Department of Commerce, Washington DC, June 1964.

[2] D. Braess, "Ueber ein Paradoxen der Verkehrsplannung", Unternehmensforschung, vol. 12, pp. 258-268, 1968.

[3] M.G.H. Bell, and Y. Iida, Transportation Network Analysis, Wiley: New York, 1997.

[4] R. B.Dial, "Minimal-revenue congestions pricing part I: A fast algorithm for the single-origin case", Transport. Res. B, Methodol., vol. B33, pp. 189-202, 1999; and references therein.

[5] R. B. Dial, "Minimal-revenue congestion pricing Part II: an efficient algorithm for the general case" Transport. Res. B, Methodol., vol. B34, no. 8, pp. 645-665, 2000.

[6] N. H. Gartner, "Optimal Traffic Assignment with elastic demands: a review. part I analysis framework" Transport. Sci., vol. 14, pp. 174-191, 1980.

[7] C. M. Penchina, "Minimal-Revenue Congestion Pricing: some more good-news and bad-news", Transp. Res. B, Methodol., vol. B38, pp. 559-570, 2004.

[8] J. G. Wardrop, "Some theoretical aspects of road traffic research", Proceedings, Institute of Civil Engineers, Part II, vol.1, pp. 325378, 1952. 
[9] C. M. Penchina and L. J. Penchina, "The Braess paradox in mechanical, traffic, and other networks", Am. J Phys, vol. 71 no. 5, pp. 479-482, May 2003.

[10] C. M. Penchina, "Power-Law Congestion Costs: Minimal Revenue (MR) pricing and the braess paradox", Open Transport. J., vol. 2, pp. 47-52, 2008.

[11] C. M. Penchina, "Braess's Paradox and Power-Law Nonlinearities in five-arc and six-arc two-terminal networks" Open Transpor. J., vol. 3 , pp. 8-14, 2009
[12] B. Calvert, and G. Keady, "Braess's paradox and power-law nonlinearities in networks" J. Austral. Math. Soc. Ser. B, vol. B35, pp. 1-22, 1993.

[13] Claude M Penchina, "Stability of Minimal Revenue Pricing", poster session 638 HP-26 (03-3501) Jan 15, 2003, in TRB 82nd Annual Meeting Sessions, 2003, Jan. 10, p. 219, and references therein to private communications from James Banks and Benjamin Heydecker, 2002.

(C) Claude M. Penchina; Licensee Bentham Open.

This is an open access article licensed under the terms of the Creative Commons Attribution Non-Commercial License (http://creativecommons.org/licenses/by$\mathrm{nc} / 3.0 /$ ) which permits unrestricted, non-commercial use, distribution and reproduction in any medium, provided the work is properly cited. 